\title{
NOTAS NOMENCLATURALES EN OPHIOGLOSSUM (OPHIOGLOSSACEAE)
}

\author{
ESTEBAN I. MEZA-TORRES ${ }^{1}$
}

\begin{abstract}
Summary: Nomenclatural notes in Ophioglossum (Ophioglossaceae). The lectotypes of Ophioglossum ellipticum Hook. \& Grev. and O. macrorrhizum Kunze are selected. The plates of protologues and herbaria specimens were compared to choose them. Discussion and illustrations of the lectotypifications are given.
\end{abstract}

Key words: Lectotype, C. S. Parker Collection, Leprier Collection, P herbarium, E herbarium.

Resumen: Se lectotipifican los nombres Ophioglossum ellipticum Hook. \& Grev. y O. macrorrizum Kunze. Para justificar la elección se compararon las ilustraciones originales y especímenes de herbario. Se discuten e ilustran las lectotipificaciones.

Palabras clave: Lectotipo, Colección C. S. Parker, Colección Leprier, Herbario P, Herbario E.

\section{INTRODUCCIÓN}

En el marco de la revisión de las Ophioglossaceae del Cono Sur y del desarrollo del Proyecto de la "Flora Argentina", y después de examinar las colecciones clásicas, surgió la necesidad de comunicar cambios en la nomenclatura y sinonimia en esta familia. Algunos de estas novedades como nuevas sinonimias en el género Ophioglossum L. (Meza-Torres et al., 2015) y lectotipificaciones en Botrychium Sw. (Meza-Torres et al., in press) ya fueron comunicados. El objetivo de esta contribución es lectotipificar los binomios de Ophioglossum ellipticum Hook. \& Grev. y $O$. macrorrhizum Kunze.

\section{TIPIFICACIONES}

Ophioglossum ellipticum Hook. \& Grev., Icon.

\footnotetext{
1 Instituto de Botánica del Nordeste, (Consejo Nacional de Investigaciones Científicas y Técnicas - Universidad Nacional del Nordeste) C.C. 209. 3400 - Corrientes, Argentina. mezatorresii@yahoo.com.ar
}

Fil. 1: 23, lám. 40, fig. A. 1831. Hab. In Demerara, Americae meridionalis; legit et communicavit amiciss. C. S. Parquer. Tipo: Guyana, Demerara, Parker s/n (Lectotypus E -E00564839!-, aquí designado. Sintypus K -K000589307!-, E -E00413859!) (= Ophioglossum nudicaule L. f.)

Esta especie fue tratada como independiente por Clausen (1938), quien consideró como ejemplar tipo a la ilustración de Hooker \& Greville (1828, tabula 40 A) (Fig. 1A). Este binomio fue puesto bajo la sinonimia de $O$. nudicaule por Wagner et al. (1984), quienes realizan un meticuloso análisis de la variabilidad de las láminas de esta especie y siguen el criterio de Clausen de tomar la ilustración mencionada como referencia.

En Kew Garden hay una cartulina depositada en la colección del herbario Hookerianum (K000589307), la cual consta de siete plantas montadas y con la inscripción "Guiana, Parker \#10". Por otro lado, en el herbario E existe otra cartulina marcada como ejemplar tipo (E00413859), la misma presenta una única planta montada y tiene la inscripción manuscrita "Demerara, Parker, 1827", ambos especímenes tienen el nombre y la cita correspondientes de O. ellipticum. Sin embargo, entre los ejemplares ordinarios del 


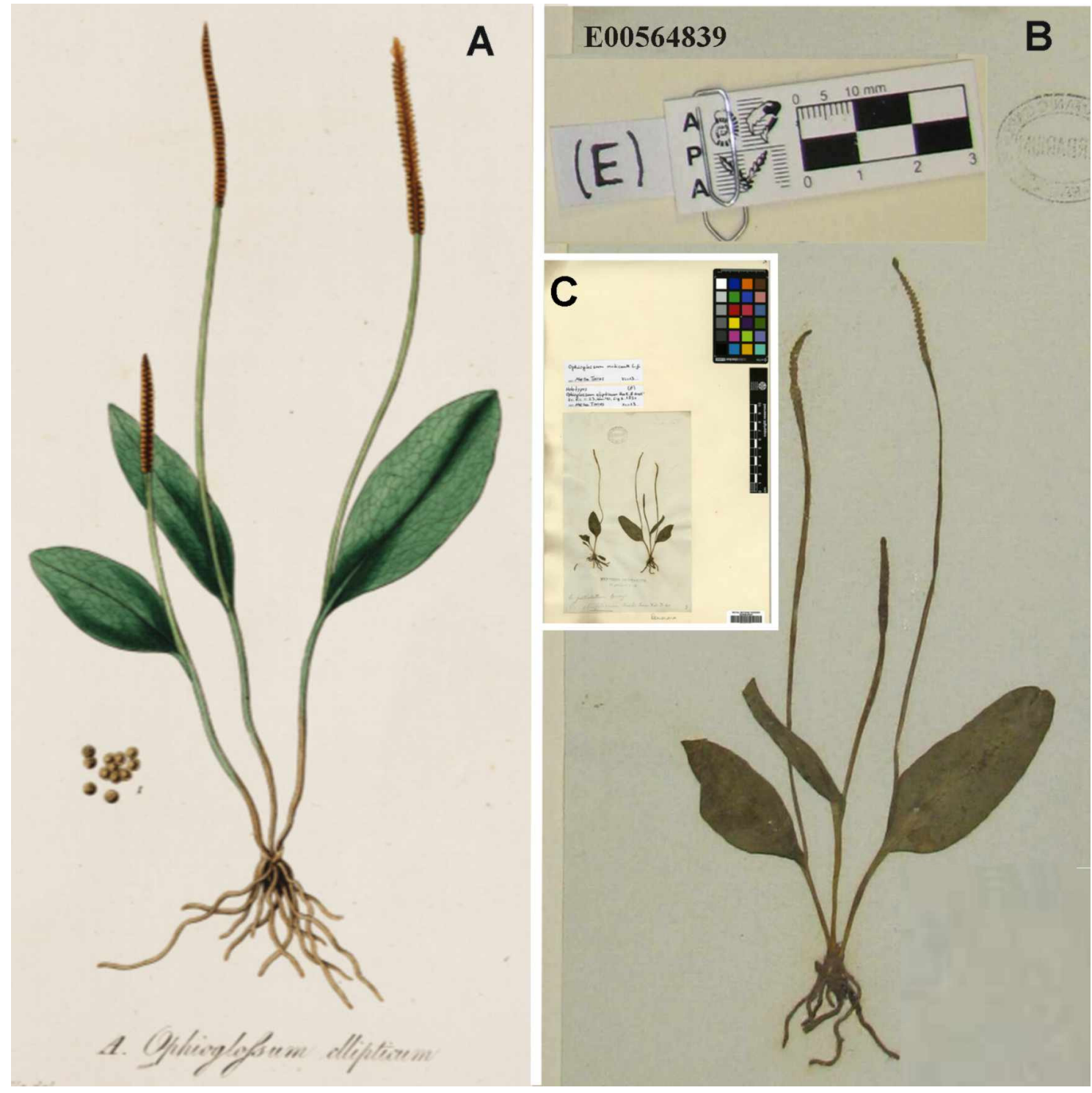

Fig. 1. Lectotipificación de Ophioglossum ellipticum. A. Tabula 40 de la publicación original de O. ellipticum (Hooker \& Greville, 1828, Icon. Fil.). B. Lectotipo y espécimen que presumiblemente fue la referencia de la ilustración original (la imagen se ha invertido con efecto espejo para su mejor comparación). C. Ejemplar completo del lectotipo de O. ellipticum. B y C: Edinburgh Herbarium (E) Royal Botanic Garden Edinburgh, Escocia.

herbario E, fue encontrada una tercer cartulina con dos plantas montadas y con el nombre y la cita manuscrita de O. ellipticum (Fig. 1C). Teniendo en cuenta que la caligrafía en las anotaciones de ese espécimen es semejante a la de Greville y que la planta montada en la parte derecha de.. la cartulina (Fig 1B) posee un fuerte paralelismo con la tabula
40 de la descripción original, es altamente probable que esa planta fuera utilizada por Greville para realizar la iconografía. Debido a que la tabula 40 de la publicación original fue utilizada por Clausen (1938) y autores posteriores para interpretar la especie, se sugiere que este último espécimen discutido, sea elegido como lectotipo. 


\section{E. I. Meza-Torres - Notas nomenclaturales en Ophioglossum}

Ophioglossum macrorrhizum, Analecta Pteridogr.: 2. 1837. Farnkräuter 1: 57 t. 29 f. 1. 1840. Tipo: In declivivis herbosis planitiae vulgo Savarmi. Flamant, ripas onessa superioris, XII1831, Leprieur 22 (Lectotypus P - P01653400! Aquí designado). Sintypus: Guiana gall, Leprier $s / n$, "Herbier Luerssen-Weigel 5624" (P -P01653392 !) (= Ophioglossum nudicaule L. f.)

En el protólogo de O. macrorrhizum se mencionan dos elementos, los cuales corresponden a dos especímenes recolectados por Leprieur, ambos depositados en el herbario P. Una cartulina pertenece al ejemplar de Leprieur \# 22 (con siete pequeñas plantas) y el otro ejemplar está incorporado a la colección Luerssen-Weigel (con tres plantas montadas). En la segunda descripción publicada por Kunze (1840) se mencionan a los mismos especímenes y se incluye una ilustración de
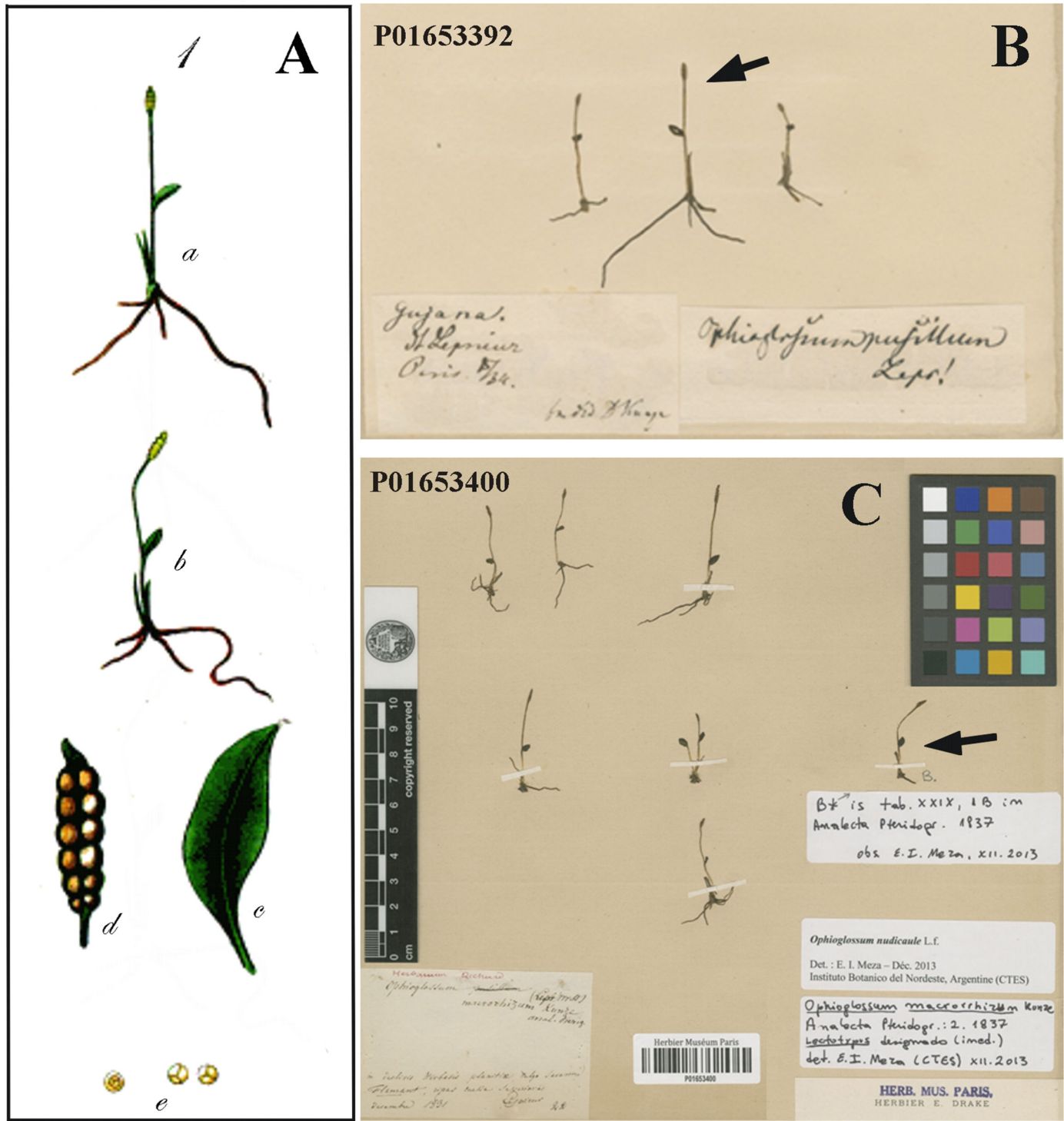

Fig. 2. Lectotipificación de Ophioglossum macrorrhizum. A. Ilustración publicada por Kunze (1840, Farnkräuter). B. Sintipo que sirvió de referencia para la ilustración original "1A" publicada por Kunze (1840). Lectotipo y espécimen que sirvió de referencia para la ilustración original "1B" publicada por Kunze (1840). B y C: ( ) Paris Herbarium (P), Muséum National d'Histoire Naturelle, (MMHN) Francia. 
dos plantas de O. macrorrhizum (Fig. 2A). Clausen (1938) al tratar este binomio, al igual que con $O$. ellipticum, considera a las ilustraciones como tipos nomenclaturales de este nombre. La ilustración de Kunze (1840) consta de dos plantas enteras y de detalles de lámina, angiostroma y esporas. La planta ilustrada en la parte superior corresponde a la planta montada en el centro de la cartulina de la colección Luerssen-Weigel (Fig. 2B), mientras que la figura inferior corresponde presumiblemente al espécimen montado cercano al margen derecho de la cartulina del ejemplar Leprieur \# 22 (2C). Esta última cartulina es elegida aquí como lectotipo por ser más abundante en el número de plantas montadas.

Si bien la elección de una ilustración como lectotipo es válida según el Código Internacional de Nomenclatura Botánica (McNeill et al., 2012) al haberse descubierto sintipos citados en los protólogos, estos deben tener prioridad para la lectotipificación (art. 9.12 y 9.19).

\section{Agradecimientos}

El autor agradece a la Myndel Botanica Foundation por el subsidio otorgado para visitar las instituciones europeas; a Jefferson Pardo, Carolina Peichoto, Silvia Ferrucci y María Mercedes Arbo por los comentarios y sugerencias; y al personal de los herbarios Royal Botanic Garden Edinburgh (Escocia) y Muséum National d'Histoire Naturelle (París).

\section{Bibliografía}

CLAUSEN, R. T. 1938. A monograph of the Ophioglossaceae. Mem. Torrey Bot. Club 19: 1-177. HOOKER, W. J. \& R. K. GREVILLE, 1828. Icon. Fil. 1. London.

KUNZE, G. 1840. Farnkräuter 1. Leipzig.

MEZA-TORRES, E. I., MACLUF, C. C., MORBELLI, M. A. \& M. S. FERRUCCCI. 2015. The circumscription of problematic species of Ophioglossum (Ophioglossaceae) from Southern South America: a palynological approach. Phytotaxa 205: 145-156.

MEZA-TORRES, E. I., STENSVOLD, M., FARRAR, D. $\&$ M. S. FERRUCCI. In press. Circumscription of the South American Moonwort Botrychium dusenii (Ophioglossaceae). Plant Biosyst.

McNEILL, J., BARRIE, F. R., BUCK, W. R., DEMOULIN, V., GREUTER, W., HAWKSWORTH, D. L., HERENDEEN, P. S., S. KNAPP, K. MARHOLD, J. PRADO, W. F. PRUD'HOMME VAN REINE, G. F. SMITH, J. H. WIERSEMA \& N. J. TURLAND. 2012. International Code of Nomenclature for algae, fungi, and plants (Melbourne Code): adopted by the Eighteenth International Botanical Congress Melbourne, Australia, July 2011. Regnum Vegetabile 157. Königstein: Koeltz Scientific Books. http:// www.iapt-taxon.org/nomen/main.php?page $=$ title

Recibido el 30 de julio de 2015, aceptado el 29 de septiembre de 2015. 